\title{
The dilemma of persisting productive or wet cough in children without established chronic lung disease: How to treat and when to investigate
}

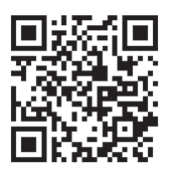

Clinicians are frequently confronted by a chronic productive or wet cough in children. A cough of more than 4 weeks is defined as chronic. It is referred to as 'productive' when a child is able to expectorate. A similar cough in younger children who are unable to expectorate, will be referred to as a 'wet' cough. The most common conditions associated with a chronic productive/wet cough are protracted bacterial bronchitis and bronchiectasis. The early diagnosis, and effective management, of a chronic productive/wet cough is therefore important. It may signal an underlying illness where early and correct management may reduce morbidity and mortality.

Chang et al. ${ }^{[1]}$ undertook two related systematic literature reviews aimed at answering two key questions (KQs) on children ( $\leq 14$ years) who present for the first time with a chronic productive/wet cough in the absence of known chronic lung disease. KQ1: How effective are antibiotics in improving resolution of cough, and if so, which antibiotic(s) and for how long? KQ2: When should they be referred for further investigation? The authors followed the CHEST expert cough panel's protocol and conducted systemic reviews of randomised controlled trials, and prospective and retrospective cohort crosssectional studies, published in English. Fifteen studies (data on 1363 children) were included in KQ1 and 17 studies (data on 2109 children) in KQ2. They concluded that:

1. There is high-quality evidence that the appropriate use of antibiotics improves cough resolution. The number needed to treat for benefit was 3 (95\% confidence interval $2.0-4.3)$.

2. There is high-quality evidence that specific cough pointers ${ }^{[2]}$ (e.g. digital clubbing, failure to thrive, cough during feeding, etc.) should prompt further investigations (e.g. flexible bronchoscopy, chest computed tomography (CT) and immunity tests).

3. There is moderate-quality evidence that children should be referred for further investigation when a productive/wet cough does not improve after 4 weeks of antibiotic treatment.

The most common bacteria reported in the studies were Haemophilus influenzae (non-typeable), Moraxella catarrhalis and Streptococcus pneumoniae. The duration of antibiotic use varied from 7 days up to 8 weeks. A 2-week antibiotic course seemed sufficient in most patients. A minority of patients received 4 weeks of antibiotic treatment. Amoxicillin-clavulanate was the most frequent choice, followed by clarithromycin. Antibiotic stewardship must remain key. More data are clearly needed on the specific criteria for antibiotic prescription in chronic wet cough. The clinical context of children presenting with a chronic productive/wet cough remains important when further investigations are ordered. These usually include chest CT, flexible bronchoscopy and appropriate laboratory investigations.

\section{André van Niekerk}

Paediatric Pulmonologist, University of Pretoria and Steve Biko Academic Hospital

\section{References}

1. Chang AB, Oppenheimer JJ, Weinberger M, Rubin BK, Irwin RS. Children with chronic wet or productive cough - treatment and investigations: A systematic review. Chest 2015; Epub. [http://dx.doi.org/10.1378/15-2065]

2. Chang AB, Glomb WB. Guidelines for evaluating chronic cough in paediatrics: ACCP Evidence-Based Clinical Practice Guidelines. Chest 2006;129(1_suppl):260S-283S. [http://dx.doi.org/10.1378/chest.129.1_suppl.260S]

S Afr Respir J 2016;22(1):26. DOI:10.7196/SARJ.2016.v22i1.61

\section{Prevention of ventilator-associated pneumonia: Is there a role for colistin nebs?}

Ventilator-associated pneumonia (VAP) is associated with a high mortality rate, increased duration of mechanical ventilation as well as additional costs. It is reassuring to note that the incidence of VAP has decreased over the last decade.

Colonisation of the lower respiratory tract, either by endogenous or exogenous pathogens, occurs rapidly after intubation. Endogenous pathogens come from contaminated oropharyngeal secretions and gastric contents. Exogenous contamination results from tracheal suctioning, fibre-optic bronchoscopy and/or ventilator circuit disconnection for nebulisations and patient transport. There is a suggestion from previous studies that there might exist a continuum from colonisation of the lower respiratory tract to the development of VAP.

Understanding the pathophysiology of VAP and improving preventive strategies has been the topic of recent research. The three broad categories of prevention include prevention of intubation itself, prevention of colonisation of the lower respiratory tract, and prevention of the progression of colonisation to development of VAP.

The use of prophylactic antibiotics to prevent VAP is an area aimed at halting progression of colonisation to the development of VAP. 
A meta-analysis by Falagas et al..$^{[2]}$ studied the use of prophylactic inhaled gentamycin, polymixin, tobramycin and ceftazadine. This study found VAP to be less frequent in those patients given prophylactic antibiotics. A recent study conducted by Karvouniaris et al. ${ }^{[3]}$ randomised 168 patients to receive inhaled colistin (500 000 units) or normal saline three times a day for 10 days or until the patient was extubated. Unfortunately, there was no significant difference in the incidence of VAP. However, there was a significantly lower incidence of VAP associated with gram-negative bacilli.

Currently, the use of prophylactic inhaled antibiotics is not recommended in the prevention of VAP. However, further studies are required in this field.

\section{Ashley C Jeevarathnum}

Paediatric Pulmonologist, University of Pretoria and Steve Biko Academic Hospital

\section{References}

1. Nseir S, Martin-Loeches I. In the name of ventilator-associated pneumonia prevention: Lung microbiota blown away by colistin! Eur Resp J 2015;46(6):15441547. [http://dx.doi.org/10.1183/13993003.01361-2015]

2. Falagas ME, Siempos II, Bliziotis IA, Michalopoulos A. Administration of antibiotics via the respiratory tract for the prevention of ICU-acquired pneumonia: A meta-analysis of comparative trials. Crit Care 2006;10(4):R123. [http://dx.doi.org/10.1186/cc5032]

3. Karvouniaris M, Makris D, Zygoulis P, et al. Nebulized colistin for ventilatorassociated pneumonia prevention. Eur Respir J 2015;46(6):1732-1739. [http://dx.doi. org/10.1183/13993003.02235-2014]

\section{Who should receive respiratory syncitial virus prophylaxis?}

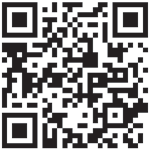

Respiratory syncitial virus (RSV) is a major cause of respiratory tract infection in both infants and children during the RSV season. Conditions predisposing to disease are chronic lung disease of prematurity, significant congenital heart disease and preterm birth $<35$ weeks' gestational age (wGA).

Palivizumab is a monoclonal antibody that has been approved for use in high-risk children and is administered at the start of and during the RSV season. Until now, use was limited to specifically defined population groups.

The Respiratory Events Among Preterm Infants Outcomes and Risk Tracking (REPORT) study conducted by Ambrose et al. ${ }^{[1]}$ addressed the excluded subgroups, which were previously perceived to be low-risk, namely the infants born 32 - 35 wGA with no associated risk factors, but as a function of chronological and gestational age. Despite $73 \%$ of all US infants being born at 32 - 35 wGA, this is the first prospective study to be conducted in the US investigating the burden of RSV disease in this population, although four similar studies have already been conducted elsewhere in the world.

Rates of RSV hospitalisation particularly from RSV emergency department (ED) visits, ICU and mechanical ventilation were similar throughout all five studies. Previously identified risk factors such as daycare attendance, preschool siblings and tobacco smoke exposure were observed. However, lower rates of RSV hospitalisations were noted among multiple birth infants.

REPORT is one of the few studies looking at RSV hospitalisation during the entire RSV season. It found that the risk of RSV ED visits and hospitalisation was highest at 6 months chronological age in the $32-34$ and 35 wGA infants with risk factors. The incidence of RSV disease severity did not differ among the $<3$ month and $3-6$ month age groups in 32 - 34 wGA infants. However, RSV illness in this group was more severe.

In light of the findings in this study, the question that needs to be answered is whether RSV prophylaxis should be provided to this population beyond 90 days of age. The elevated RSV hospitalisation rates among infants 35 wGA with the 2012 American Academy of Paediatrics environmental risk factors at $<3$ months and $3-<6$ months also requires review.

\section{Denise C Parris}

Fellow, Paediatric Pulmonology University of Pretoria and Steve Biko Academic Hospital

\section{Reference}

1. Ambrose CS, Anderson EJ, Simões AF, et al. Respiratory syncytial virus disease in preterm infants in the U.S. born at 32-35 weeks gestation not receiving immunoprophylaxis. Pediatr Infect Dis J 2014;33(6):576-582. [http://dx.doi. org/10.1097/INF.0000000000000219]

\section{Early inhaled budesonide for the prevention of broncho- pulmonary dysplasia}

Although with modern methods of prevention and management of prematurity, bronchopulmonary dysplasia (BPD) is a less common consequence of being born prematurely, the 'new' form of BPD is still a debilitating and difficult-to-manage problem. Attempts to prevent it, or manage it early, are quite disappointing. Systemic steroids given to extremely premature infants with respiratory distress do reduce the incidence of BPD but have important side-effects including compromising brain development. Use of inhaled steroids has documented inconsistent findings.

Bassler et al. ${ }^{[1]}$ randomly assigned 863 premature infants to early (within 24 hours of birth) inhaled budesonide or placebo and 
continued this therapy until they were no longer on oxygen or reached a postnatal age of 32 weeks. BPD was assessed at 36 weeks by means of standardised oxygen-saturation monitoring.

The incidence of BPD was $27.8 \%$ in the budesonide group versus $38.0 \%$ in the placebo group (relative risk (RR) $0.86,95 \%$ confidence interval (CI) $0.75-1.00, p=0.05)$. Death occurred in $16.9 \%$ and $13.6 \%$ of the patients, respectively (RR $1.24,95 \%$ CI $0.91-1.69$, $p=0.17$ ).

The authors conclude that among extremely premature infants, the incidence of BPD was lower among those who received early inhaled budesonide than in those receiving placebo. However, they go on to point out that the advantage may come at the expense of increased mortality.

While seeking a solution to the devastating effects of BPD on children, it seems unlikely that this study will motivate the widespread use of inhaled steroids. However, it does suggest that inhaled steroids may have a role. It would be interesting to repeat this study using newer steroids such as fluticasone, mometasone or ciclesonide, which theoretically have lower systemic effects.

\section{Robin J Green}

Paediatric Pulmonologist, University of Pretoria and Steve Biko Academic Hospital

\section{Reference}

1. Bassler D, Plavka R, Shinwell ES, et al. Early inhaled budesonide for the prevention of bronchopulmonary dysplasia. N Eng J Med 2015;373:1497-1506. [http://dx.doi. org/10.1056/NEJMoa1501917]

S Afr Respir J 2016;22(1):27-28. DOI:10.7196/SARJ.2016.v22i1.64 